\title{
Avaliação da utilização de dentifrício fluoretado em crianças
}

\author{
Fluoride toothpaste use evaluation in children
}

\author{
Aline Cardoso de Jagher \\ Tamara Ripplinger** \\ Gabriela dos Santos Pinto \\ Lisandrea Rocha Schardosim ${ }^{* * * *}$
}

\section{Resumo}

Introdução: o uso de dentifrício fluoretado é uma das principais formas de prevenção e controle da doença cárie em crianças. Objetivo: avaliar a utilização de dentifrícios fluoretados por crianças que procuraram atendimento odontológico em uma instituição de ensino superior. Sujeitos e método: este estudo transversal envolveu pais/responsáveis e crianças que procuraram atendimento nas Unidades de Clínica Infantil da Faculdade de Odontologia da Universidade Federal de Pelotas, no período de 14 a 29 de janeiro de 2014. Foi realizada uma entrevista com o responsável para a coleta de dados demográficos e socioeconômicos, hábitos de higiene e visitas odontológicas prévias. Foi mostrado um folheto ilustrativo sobre as quantidades de dentifrício e solicitou-se ao responsável que colocasse o dentifrício na escova. As escovas foram pesadas em balança de precisão. Os dados foram analisados no programa Stata 10.0. Resultados: participaram do estudo 87 crianças, com idades entre 3 e 12 anos, das quais 50 (56,8\%) frequentavam regularmente a instituição. $O$ dentifrício fluoretado na concentração adequada era usado por 82 crianças (96,5\%) e foi introduzido antes de 1 ano de idade em 27 delas $(31,8 \%)$. Sessenta e oito por cento das crianças era responsável pela própria escovação e 73,6\% pela colocação de dentifrício na escova. Conclusões: os responsáveis desconheciam a idade correta para introdução do dentifrício e os riscos de fluorose. Os achados sugerem que mais esforços devem ser empregados na orientação do correto uso de dentifrícios fluoretados por crianças, principalmente em ambiente acadêmico.

Palavras-chave: Criança. Dentifrícios. Escovação dentária. Flúor.

\section{Introdução}

A cárie é uma doença multifatorial e, apesar de sua prevalência estar declinando nas diferentes faixas etárias, é considerada um relevante problema de saúde pública ${ }^{1}$. Conforme dados do Ministério da Saúde, no Brasil, aos 5 anos de idade, uma criança possui em média 2,43 dentes com experiência de cárie, sendo que o componente cariado é responsável por mais de $80 \%$ do índice e é a maior causa de dor dental, podendo afetar o crescimento e interferir na qualidade de vida das crianças ${ }^{2,3}$. Atualmente, uma das formas mais amplas da utilização do flúor é o emprego de dentifrícios fluoretados, sendo a substância mais importante presente no creme dental, aumentando o efeito de escovação mecânica sobre o controle da cárie dentária ${ }^{4}$.

Os dentifrícios fluoretados contribuem aproximadamente com $57 \%$ da quantidade de flúor ingerida diariamente por crianças de 4 a 6 anos de idade. Isso acontece especialmente em pré-escolares, devido à inabilidade dessas crianças cuspirem todo o dentifrício ${ }^{5}$. Portanto, algumas medidas são necessárias para controlar a ingestão de fluoreto, como optar por aqueles que não seduzem a criança pelo sabor e diminuir a quantidade aplicada na escova dental ${ }^{6}$.

Pendrys et al. ${ }^{7}$ (2010) verificaram uma pequena relação entre o modo que algumas crianças realizavam a escovação e a incidência de fluorose. Constataram que as crianças que colocavam dentifrício na metade ou mais das cerdas da escova apresentavam frequências mais elevadas de fluorose leve. Martins

\footnotetext{
Acadêmica da Faculdade de Odontologia, Universidade Federal de Pelotas, Pelotas, Rio Grande do Sul, Brasil.

Aluna de mestrado em Odontopediatria do Programa de Pós-Graduação em Odontologia da Universidade Federal de Pelotas, Pelotas, Rio Grande do Sul, Brasil.

** Professora substituta das Unidades de Saúde Bucal Coletiva, Departamento de Odontologia Social e Preventiva, Faculdade de Odontologia, Universidade Federal de Pelotas, Pelotas, Rio Grande do Sul, Brasil.

* Professora adjunta das Unidades de Clínica Infantil, Departamento de Odontologia Social e Preventiva, Faculdade de Odontologia, Universidade Federal de Pelotas, Pelotas, Rio Grande do Sul, Brasil.
} 
et al. ${ }^{8}$ (2011) avaliaram se os fatores socioeconômicos influenciavam na escolha de dentifrícios comercializados e se esses produtos estavam associados à maior ingestão de flúor $(\mathrm{F})$. Os autores constataram que a prática de colocar dentifrício fluoretado em toda a extensão das cerdas foi associada ao nível socioeconômico médio e baixo e à baixa escolaridade dos pais.

Com base na melhor evidência científica disponível sobre estratégias para o controle de cárie, recomenda-se, atualmente, que um dentifrício fluoretado de, no mínimo, 1100 ppm de flúor seja utilizado duas vezes ao dia como coadjuvante da limpeza dos dentes de todas as crianças, principalmente direcionada aos dentes com alto risco de cárie, tais como os primeiros molares decíduos e permanentes em erupção $0^{4,9}$. A Associação Gaúcha de Odontopediatria recomenda também que, enquanto a criança não tiver condições de se autocuidar, o uso de dentifrício fluoretado seja de responsabilidade dos pais ou cuidadores, garantindo assim maior segurança quanto à fluorose ${ }^{9}$.

Tendo em vista as novas orientações da comunidade científica a respeito do uso de dentifrícios fluoretados, a avaliação de seu emprego por crianças torna-se um objeto de estudo interessante para indicar se as recomendações estão sendo seguidas. Assim, este estudo teve como objetivo avaliar a utilização de dentifrícios fluoretados pelas crianças que procuraram atendimento odontológico na Unidade de Clínica Infantil da Faculdade de Odontologia da Universidade Federal de Pelotas.

\section{Sujeitos e método}

Este estudo teve aprovação do Comitê de Ética em Pesquisa da Faculdade de Odontologia da Universidade Federal de Pelotas (Parecer nº 68/2013). Os pais e/ou cuidadores/responsáveis foram esclarecidos acerca dos objetivos da pesquisa e assinaram o Termo de Consentimento Livre e Esclarecido e a criança foi incluída mediante assinatura do Assentimento Livre e Esclarecido.

A pesquisa caracterizou-se como um estudo transversal que envolveu pais/responsáveis e crianças que procuraram atendimento nas Unidades de Clínica Infantil (UCI) da Faculdade de Odontologia da Universidade Federal de Pelotas (FO-UFPel) no período de 14 a 29 de janeiro de 2014, tanto para atendimento de rotina como de urgência, e aquelas que compareceram no dia da triagem, para oferta de novas vagas na unidade, realizada no dia 19 de março de 2014.

Previamente ao início da coleta de dados, foi realizado um treinamento para adequação do roteiro de entrevista e pesagem das escovas. Duas acadêmicas de odontologia treinadas auxiliaram na pesquisa.

Foram incluídas todas as crianças que procuraram o serviço, independente da faixa etária, desde que acompanhadas de um dos pais ou responsável que conhecesse a rotina da criança e fosse capaz de responder às questões do estudo. As crianças que não vieram acompanhadas dos pais ou de um responsável legal e aquelas cujos responsáveis não consentissem a pesquisa foram excluídas do estudo.

Inicialmente, foi realizada uma entrevista com o responsável a fim de coletar dados demográficos e socioeconômicos, tais como idade, sexo, escolaridade materna, renda da família, estrutura familiar (nuclear: criança que vive com pai e mãe / não nuclear: criança que vive sem o pai, a mãe ou sem ambos), hábitos de higiene e visitas odontológicas prévias. Após a entrevista, foi mostrado um folheto ilustrativo (Figura 1), contendo fotos das quantidades de dentifrício (grão de ervilha, técnica transversal e técnica longitudinal), a fim de que o responsável por sua colocação (criança ou responsável) indicasse a foto que melhor representasse a quantidade normalmente colocada.

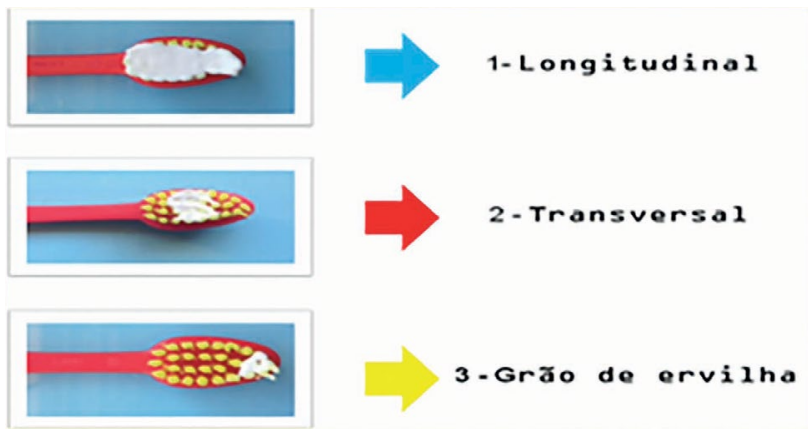

Figura 1 - Folheto com as quantidades de dentifrício (grão de ervilha, técnica transversal e técnica longitudinal)

Fonte: elaboração dos autores.

Posteriormente, foi solicitado ao responsável que dispusesse o dentifrício oferecido sobre uma escova fornecida pelos pesquisadores. Então, realizou-se a pesagem dessas para a verificação da quantidade de dentifrício colocada, utilizando uma Balança Analítica de Precisão Bel Mark 210. Os dados foram registrados em uma ficha específica. A quantidade de dentifrício real foi obtida por pesagem e categorizada da seguinte forma: grão de arroz cru: $\cong 0,1 \mathrm{~g}$; grão de ervilha: $\geq 0,2 \leq 0,3$ g; técnica transversal: $\geq 0,31 \mathrm{~g}$ a $\leq 0,90 \mathrm{~g}$; técnica longitudinal: $\geq 0,91 \mathrm{~g}$. Considerou-se uso adequado de dentifrício quando crianças menores de 2 anos utilizavam a quantidade referente a um grão de arroz cru, de 3 a 5 anos a quantidade de um grão de ervilha e a partir dos 6 anos, técnica transversal.

As informações dos questionários e da pesagem das escovas foram digitadas em um banco de dados do programa Microsoft Excel e analisadas com o auxílio do programa Stata versão 10.0. Os dados foram submetidos à análise estatística descritiva e apresentados em forma de frequência relativa e seus percentuais. O teste Qui-Quadrado foi utilizado para avaliar as possíveis associações entre as variáveis, com nível de significância de $5 \%$. 


\section{Resulltados}

Foram entrevistados responsáveis de 87 crianças entre 3 e 12 anos de idade, com média de idade de 7,8 anos $(\mathrm{DP}=2,3)$, das quais $50(56,8 \%)$ frequentavam regularmente a UCI da FO-UFPel e 37 $(37,9 \%)$ não frequentavam. Dentre essas, $30(30 \%)$ aguardavam o exame de triagem para ingresso no serviço e $7(7,9 \%)$ aguardavam atendimento de urgência. Em relação ao sexo, 41 pertenciam ao masculino $(47,1 \%)$ e 46 ao feminino $(52,8 \%)$. A Tabela 1 apresenta a distribuição da amostra segundo as características estudadas nesta pesquisa.

Tabela 1 -Distribuição da amostra segundo características estudadas, Pelotas, $2014(n=87)$

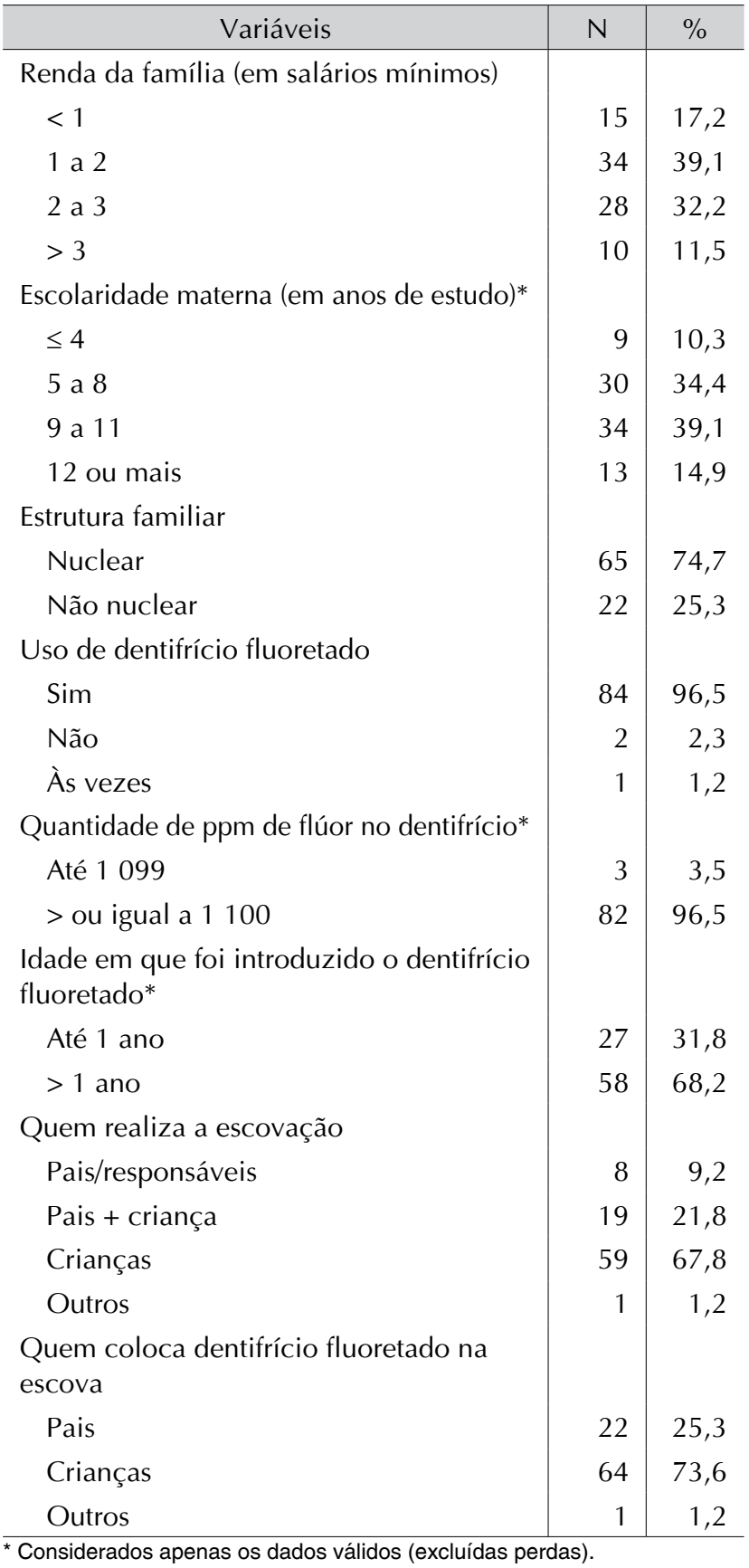

Fonte: dados da pesquisa.
Com relação ao tipo de escova utilizada pelas crianças, 57 delas $(65,5 \%)$ costumavam usar escova modelo infantil e $30(34,5 \%)$ usavam modelo adulto. No que se refere à frequência de escovação diária, 64 dos entrevistados $(73,6 \%)$ afirmaram que seus filhos costumavam escovar os dentes 3 vezes ou mais por dia, $20(23 \%)$ apontaram 2 vezes ao dia, e 3 pais $(3,5 \%)$ relataram que os filhos escovam os dentes 1 vez ao dia.

Foi questionado se as crianças sabiam cuspir o dentifrício durante a escovação e verificou-se que 73 entrevistados $(83,9 \%)$ relataram que a criança sabia cuspir, dez $(11,5 \%)$ relataram que ela não sabia e um $(1,2 \%)$ não sabia informar. A Figura 2 apresenta a relação entre a quantidade de dentifrício referida (apontada no folheto) e a quantidade real colocada (obtida por pesagem).

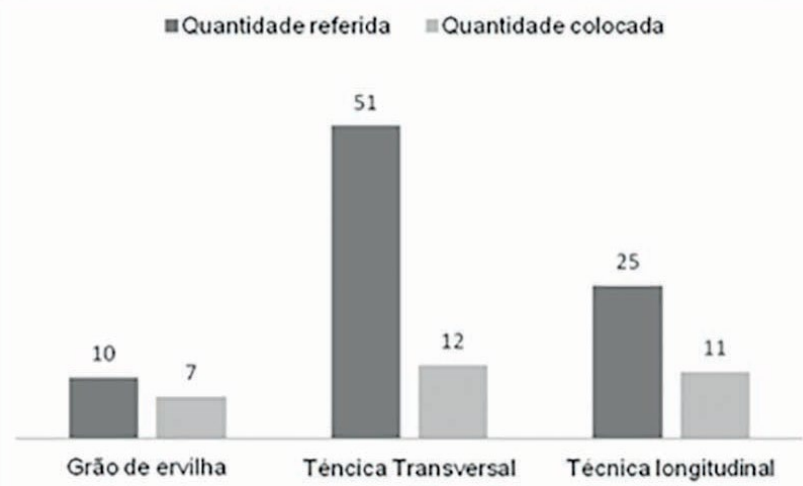

Figura 2 - Distribuição de quem coloca dentifrício na escova de acordo com a quantidade referida (indicada na foto) e a quantidade real colocada na escova, Pelotas, $2014(n=86)$

Fonte: dados da pesquisa.

Em relação à orientação recebida de um profissional sobre o uso de dentifrício fluoretado, observou-se que 53 crianças $(63,1 \%)$ haviam recebido orientação e $30(35,7 \%)$ declararam não ter recebido. A Tabela 2 apresenta a relação entre ter recebido orientação sobre a quantidade de dentifrício a ser colocada na escova e ter consultado um cirurgião-dentista. Não foi observada associação estatisticamente significativa entre consultar um cirurgião-dentista e receber orientação sobre uso de dentifrício fluoretado.

Tabela 2 - Distribuição da amostra de acordo com consulta a um cirurgião-dentista e orientação sobre utilização de dentifrício na escovação, Pelotas, $2014(n=83)$

\begin{tabular}{|c|c|c|c|c|c|c|}
\hline \multirow[b]{3}{*}{$\begin{array}{l}\text { Consulta ao } \\
\text { cirurgião-dentista* }\end{array}$} & \multicolumn{6}{|c|}{$\begin{array}{l}\text { Recebeu orientação sobre uso de } \\
\text { dentifrício fluoretado* }\end{array}$} \\
\hline & \multicolumn{2}{|c|}{ Sim } & \multicolumn{2}{|c|}{ Não } & \multicolumn{2}{|c|}{ Total } \\
\hline & $n$ & $\%$ & $\mathrm{n}$ & $\%$ & $n$ & $\%$ \\
\hline Sim & 45 & 64,3 & 25 & 35,7 & 70 & 100 \\
\hline Não & 8 & 61,5 & 5 & 38,5 & 13 & 100 \\
\hline
\end{tabular}


Os responsáveis foram questionados sobre a importância da utilização de flúor no dentifrício, e 72 deles $(86,8 \%)$ relataram ser importante. Dentre os 87 responsáveis, 28 (29,9\%) declararam que o flúor é importante pelo efeito protetor contra as cáries, $22(25,3 \%)$ responderam que melhora a limpeza dos dentes, $9(10,3 \%)$ consideraram importante, mas não sabiam justificar, e 26 responsáveis $(29,9 \%)$ não sabiam informar.

A Tabela 3 apresenta a distribuição dos responsáveis segundo atendimento regular na UCI da FO-UFPel e uso adequado de dentifrício fluoretado pelas crianças. Não foi observada associação estatisticamente significativa entre ser atendido regularmente na UCI e usar de forma adequada dentifrício. Em relação à quantidade de dentifrício, observou-se que 45 crianças $(53,6 \%)$ usavam a quantidade recomendada e $39(46,4 \%)$ não usavam.

Tabela 3 - Distribuição da amostra segundo atendimento regular na Unidade de Clínica Infantil da FO-UFPel e uso adequado de dentifrício fluoretado, Pelotas, $2014(n=84)$

\begin{tabular}{l|c|c|c|c|c|c} 
& \multicolumn{4}{c}{ Uso adequado de dentifrício* } \\
\cline { 2 - 7 } & \multicolumn{2}{c}{ Sim } & \multicolumn{2}{c}{ Não } & \multicolumn{2}{c}{ Total } \\
\hline $\begin{array}{l}\text { Atendimento } \\
\text { regular na UCI* }\end{array}$ & $\mathrm{n}$ & $\%$ & $\mathrm{n}$ & $\%$ & $\mathrm{n}$ & $\%$ \\
\hline Sim & 27 & 56,3 & 21 & 43,7 & 48 & 100 \\
Não & 18 & 50,0 & 18 & 50,0 & 36 & 100 \\
\hline
\end{tabular}

* Considerados apenas os dados válidos (excluídas perdas); teste Qui-Quadrado $(p<0,05)$.

Fonte: dados da pesquisa.

\section{Discussão}

O flúor dinamicamente importante é aquele que está presente na cavidade bucal e participa diretamente dos processos de desmineralização e remineralização (DES/RE) da superfície dental, durante todo o processo da doença cárie, e não apenas pela incorporação de flúor em baixa concentração no esmalte. O flúor proveniente do dentifrício é tão abrangente quanto a água fluoretada em termos de saúde pública e em relação ao seu possível potencial de efeito cariostático ${ }^{10}$. A recomendação atual é a de que o dentifrício fluoretado seja empregado em todas as faixas etárias, a partir do primeiro dente decíduo ${ }^{4,9}$. Embora não esteja padronizada a quantidade exata de dentifrício a ser depositada na escova, estudos demonstraram que pequenas doses (cerca de 0,3 g) devem ser administradas por crianças menores de 7 anos de idade ${ }^{4,9}$.

Dessa forma, avaliar se essas recomendações estão sendo colocadas em prática é fundamental, principalmente considerando crianças que frequentam uma unidade de ensino. Nesse contexto, os achados demonstraram que quase a totalidade das crianças que participaram deste estudo utilizavam dentifrício fluoretado de forma adequada, com a concentração mínima de 1100 ppm de flúor. Entretanto, apenas $31,8 \%$ dos pais/responsáveis relataram introduzir o dentifrício fluoretado antes de um ano de idade, fase em que ocorre a erupção dos primeiros dentes decíduos. O resultado encontrado coincide com relatos da literatura de que a exposição ao dentifrício fluoretado, em algumas crianças, ocorre antes de um ano de vida ${ }^{11}$.

Outra preocupação levantada nesta pesquisa está relacionada com o fato de $70 \%$ das crianças serem as responsáveis pela sua escovação. Crianças menores de 6 anos não têm destreza manual suficiente e coordenação motora para realizar uma higiene bucal efetiva, acarretando em uma limpeza insuficiente dos dentes e em maior risco de doenças bucais. Os pais devem ser orientados a realizar a escovação até a criança completar 3 anos de idade, solicitando o auxílio da criança depois de os dentes estarem limpos, a fim de familiarizá-la e comprometê-la com os cuidados bucais. Dos 3 aos 6 anos, as crianças começam a ter mais habilidade, entretanto, a maior responsabilidade ainda é dos pais. A partir dos 6 anos, a higiene bucal pode ser realizada pela criança, porém com a supervisão dos pais ${ }^{12-14}$.

Pelo fato de a maioria das crianças da pesquisa ter o costume de realizar sua própria higiene bucal, um grande número delas também coloca o dentifrício na escova. Essa constatação é preocupante, pois a tendência das crianças, normalmente, é colocar dentifrício em grandes quantidades, principalmente se os dentifrícios tiverem sabor agradável.

Quando se enfatiza a questão da supervisão das crianças durante a escovação, associa-se essa medida ao fato de que crianças pequenas não apresentam capacidade de cuspir todo o dentifrício, pois é sabido que as crianças menores apresentam dificuldades motoras em controlar a deglutição, fazendo com que não consigam expelir toda a saliva com o dentifrício. Segundo o relato dos responsáveis, dez crianças ainda não tinham desenvolvido essa habilidade. A recomendação de um responsável dispensar o dentifrício na escova é uma medida preventiva que visa evitar o risco de fluorose dental ${ }^{12-14}$.

A observação dos dados demonstrou que mais de $70 \%$ dos responsáveis relataram que a higiene bucal é realizada três vezes ao dia ou mais, fato que poderia demonstrar que os pais estão empenhados na saúde bucal de seus filhos. Entretanto, é necessário lembrar que ao serem entrevistados os responsáveis podem ter respondido adequadamente por julgar que seria a resposta correta. Martins et al. ${ }^{8}$ (2011) relatam que os pais podem ter um comportamento mais cuidadoso durante a entrevista por estarem sendo submetidos a uma pesquisa.

Segundo Damião et al. ${ }^{14}$ (2010), a maioria dos pais ou responsáveis relatou utilizar uma pequena quantidade de dentifrício fluoretado. No entanto, no presente estudo, observou-se que a quantidade colocada na escova foi superior à recomendada para a faixa etária, pois a dose referida pelo responsável 
ou pela criança foi menor que a colocada na escova. Acredita-se que pais e crianças tenham dificuldades em assimilar as orientações oferecidas apenas verbalmente pelos profissionais. Com isso, destaca-se a importância de que os cirurgiões-dentistas instruam seus pacientes e familiares não apenas com palavras, mas encontrem métodos alternativos como ilustrações, demonstrações visuais das quantidades apropriadas para cada faixa etária.

De acordo com Pacca et al..$^{15}$ (2003), que avaliaram estudantes de odontologia, apesar de todos os esforços preventivos que os alunos ofereciam aos pacientes, o caráter humanístico da profissão careceu maior desenvolvimento. Isso pode refletir na despreocupação em saber se o paciente realmente compreende as orientações oferecidas pelo profissional.

Os resultados encontrados neste estudo revelaram que a consulta prévia a um cirurgião-dentista não determinou ter recebido orientação acerca do uso adequado de dentifrício, pois mais de $80 \%$ dos pais que relataram ter levado a criança a um profissional afirmaram que não receberam essa informação. Esse dado concorda com os resultados apresentados por Valois et al. ${ }^{11}$ (1999), os quais indicaram que apenas $20,8 \%$ dos pais tinham sido orientados sobre a quantidade ideal de dentifrício a utilizar.

Da mesma forma, ser paciente e frequentar regularmente a Unidade de Clínica Infantil da FO-UFPel não foi associado ao uso adequado de dentifrício. Pelo contrário, mais de $50 \%$ das crianças que colocam mais dentifrício na escova do que o recomendado frequentam a clínica. Essa situação é preocupante, pois se esperava que, por ser um ambiente acadêmico, orientações adequadas estivessem sendo oferecidas. Frente a essa situação, torna-se importante encontrar estratégias de educação em saúde efetivas, a fim de melhorar os resultados encontrados além de padronizar orientações e condutas preventivas entre os acadêmicos.

Em relação ao conhecimento dos responsáveis sobre o flúor, verificou-se que a maioria deles $(86,8 \%)$ acredita que o flúor no dentifrício é importante, porém não sabe o motivo, pois apenas $29,9 \%$ declararam que o flúor é importante pelo efeito protetor contra as cáries.

\section{Conclusões}

Apesar de a maioria das crianças envolvidas no estudo utilizar dentifrícios fluoretados na dose e concentração adequadas, os responsáveis desconheciam a idade correta para introdução do dentifrício na higiene e os riscos de desenvolvimento de fluorose, pois, em grande parte da amostra, as crianças eram responsáveis pela manipulação do dentifrício e pela sua própria escovação. Os achados deste estudo sugerem que mais esforços devem ser empregados na orientação do correto uso de dentifrícios fluoretados por crianças, principalmente em ambiente acadêmico.

\section{Abstract}

Introduction: Fluoride toothpaste is one of the main forms of prevention and control of caries in children. Objective: To assess the use of fluoride toothpastes by children who sought dental care at a higher education institution. Subjets and method: This cross-sectional study involved parents/guardians and children who sought care in Child Clinic Units of the School of Dentistry, Federal University of Pelotas, RS, Brazil, from January 14 to 19, 2014. An interview was conducted with the guardians of children to collect demographic and socioeconomic data, hygiene habits, and previous dental visits. An illustrative brochure about the quantities of toothpaste was shown, and the guardian was asked to apply toothpaste on the toothbrush. The brushes were weighed on a precision scale. Data were analyzed in the Stata 10.0 software. Results: The study included 87 children, aged between 3 and 12 years, from which 50 (56.8\%) regularly attended the institution. Eighty-two children $(96.5 \%)$ used the appropriate concentration of fluoride toothpaste, which was introduced before 1 year old to 27 of them (31.8\%). Sixty-eight percent of children was responsible for their own toothbrushing and $73.6 \%$ for applying toothpaste on the toothbrush. Conclusions: Guardians were unaware of the proper age to introduce toothpaste and of the risks of developing fluorosis. The findings suggest that further efforts should be applied for instructing about the proper use of fluoride toothpastes by children, especially in the academic environment.

Keywords: Child. Fluoride. Toothbrushing. Toothpastes.

\section{Referências}

1. Ministério da Saúde. Projeto SB Brasil 2010. [citado 2013 set. 25]. Disponível em URL: http://189.28.128.100/dab/ docs/geral/apresentacao_SB2010.pdf.

2. Goettems ML, Ardenghi TM, Romano AR, Demarco FF, Torriane DD. Influence of maternal dental anxiety on oral health-related quality of life of preschool children. Qual Life Res 2010; 20(6):951-9.

3. Boeira GF, Correa MB, Peres KG, Peres MA, Santos IS, Matijasevich A, et al. Caries is the main cause for dental pain in childhood: findings from a birth cohort. Caries Res 2012; 46(5):488-95.

4. Cury JA, Tenuta LMA. Evidence-based recommendation on toothpaste use. Braz Oral Res 2013; 12(1):1-7.

5. Pessan JP, Silva SMB, Buzalaf MAR. Evaluation of the total fluoride intake of 4-7-year-old children from diet and dentifrice. J Appl Oral Sci 2003; 11(2):150-6.

6. Davies RM, Ellwood RP, Davies GM. The rational use of fluoride toothpaste. Int J Dent Hyg 2003; 1(1):3-8.

7. Pendrys DG, Haugejorden O, Bardsen A, Wang NJ, Gustavsen F. The risk of enamel fluorosis and caries among Norwegian children: implications for Norway and the United States. J Am Dent Assoc 2010; 141(4):401-14.

8. Martins CC, Oliveira MJ, Pordeus IA, Cury JA, Paiva SM. Association between socioeconomic factors and the choice of dentifrice and fluoride intake by children. Public Health Res Pract 2011; 8(11):4284-99. 
9. Agoped - Associação Gaúcha de Odontopediatria. Dentifrícios fluoretados na primeira infância: riscos e benefícios [homepage na internet]. [citado 2013 set. 25]. Disponível em URL: http://www.agoped.org.br/carta.pdf.

10. Cury JA, Hashizume LN, Del Bel Cury AA, Tabchoury CPM. Effect of dentifrice containing fluoride and/or baking soda on enamel demineralization/remineralization: an in situ study. Caries Res 2001; 35(2):106-10.

11. Valois CA, Soviero VM, Cruz RA. Avaliação do grau de fluorose em crianças de 7 a 12 anos de idade. J Bras Odontoped Odonto Bebe 1999; 2(9):383-90.

12. Polk DE, Geng M, Levy S, Koerber A, Flay BR. Frequency of daily tooth brushing: predictors of change in 9- to11-year old US children. Community Dent Health 2014; 31(3):136-40.

13. Zeedyka MS, Longbottomb C, Pittsb NB. Tooth-Brushing Practices of Parents and Toddlers: A Study of Home-Based Videotaped Sessions. Caries Res 2005; 39(1):27-33.

14. Damião LS, Abrão LSO, Lopes PP, Castro AM, Oliveira FS. Uso de dentifrício fluoretado e hábitos de escovação em crianças de 6 a 36 meses de idade. Rev Odontol Bras Central 2010; 19(51):295-300.

15. Pacca S, Corrêa L, Motta M. Auto-imagem do cirurgião-dentista: um estudo baseado em desenhos de alunos de graduação. Rev Abeno 2003; 3(1):82-5.

\section{Endereço para correspondência:}

Lisandrea Rocha Schardosim

Rua Gonçalves Chaves, 457

Bairro Centro

96015-560 Pelotas, RS

Telefone: (53) 3225-6741 Ramal 124

E-mail: lisandrears@hotmail.com

Recebido: 05/10/15. Aceito: 11/03/16. 